\title{
Comunidad de indagación como ambiente de aprendizaje: una propuesta y una apuesta
}

\author{
Iván Darío Cruz Vargas \\ Lizeth Ximena Castro Patarroyo ${ }^{2}$ \\ Manuel Alejandro Ojeda Suárez ${ }^{3}$
}

\begin{abstract}
1.Universidad Pedagógica y Tecnológica de Colombia, Colombia ivancruzv93@gmail.com

2.Universidad Pedagógica y Tecnológica de Colombia, Colombia

3. Institución Educativa Departamental Tierra de Promisión, Colombia

Como citar: Cruz, I., Castro Patarroyo, L., \& Ojeda Suárez, M. (2020). Comunidad de indagación como ambiente de aprendizaje: una propuesta y una apuesta. EDUCACIÓN Y CIENCIA, (24), e11404. https://doi. org/10.19053/0120-7105.eyc.2020.24. e11404
\end{abstract}

\section{Resumen}

Este artículo presenta los resultados de investigación relacionados al proyecto: Filosofía para/con Niños $(\mathrm{Fp} / \mathrm{cN})$ como un estudio de innovación educativa en el desarrollo del pensamiento científico en instituciones educativas rurales del departamento de Boyacá. La propuesta parte de una construcción teórica del ambiente de aprendizaje y la configuración de éste desde la perspectiva del programa Filosofía para Niños (FpN) de Mathew Lipman, y las apropiaciones referentes a Filosofía con Niños ( $\mathrm{FcN})$. Así mismo, con el objetivo de diseñar y desarrollar un ambiente de aprendizaje creado en clave de comunidad de indagación, el estudio tuvo en cuenta diferentes experiencias documentadas que permitieron la aplicación y evaluación a manera de estudio de caso, de una propuesta desarrollada en el área de Ética y Valores Humanos con estudiantes de grado sexto de una institución educativa de la ciudad de Tunja. Se concluye con las aportaciones y pertinencia de la comunidad de indagación como ambiente de aprendizaje, la cual, se plantea como una posibilidad de transformación curricular de la institucionalidad educativa.

Palabra clave: ambientes de aprendizaje, didáctica, enseñanza de la filosofía.

Recibido: $14 / 03 / 2020$ | Revisado: $31 / 05 / 2020$

Aprobado: 25/07/2020 | Publicado: 18/08/2020 


\title{
Comunidad de indagación como ambiente de aprendizaje: una propuesta y una apuesta
}

\begin{abstract}
This article presents the results of Philosophy for / with Children (Fp / $\mathrm{cN}$ ) as a study of educational innovation in the development of scientific thinking in rural educational institutions in the department of Boyacá, project. The proposal begins with a theoretical construction of the learning environment and a configuration of it from the perspective of the program Philosophy for Children $(\mathrm{FpN})$ of the author Mathew Lipman, and the diverse adaptations to the Philosophy with Children $(\mathrm{FcN})$, likewise, with the aim of designing and developing a learning environment created in the key of an inquiry community, the study took into account different documented experiences that allowed the application and evaluation as a case study of a proposal developed in the area of Ethics and Human Values with sixth grade students from an educational institution in the city of Tunja. The text concludes make evident the contributions and relevance of the community of inquiry as a learning environment, hence, proposed as a possibility of curricular transformation of educational institutions
\end{abstract}

Keyword: learning environments, didactics, philosophy teaching

\section{Introducción}

Debido a las transformaciones en el ámbito político, económico y tecnológico que ha implicado la globalización, se acusa a la educación y su institucionalidad de continuar prácticas rezagadas y desactualizadas. Por ello, se hace necesario reflexionar sobre los recursos, materiales, espacios, tiempos, relaciones intersubjetivas y el proyecto de sujeto en el marco social de la escuela, para de esta manera, plantear nuevas formas de proceder que involucren e impacten la institucionalidad educativa. En otras palabras, los escenarios de educación como instituciones de agenciamiento cultural, deben cuestionar y resignificar los ambientes de aprendizaje en los cuales se desenvuelven. En este sentido y con el ánimo de aportar a la comprensión y consolidación de la comunidad de indagación como ambiente de aprendizaje, el articulo presenta los resultados de investigación del proyecto titulado Filosofía para/con niños como innovación educativa en el desarrollo del pensamiento científico en Instituciones educativas rurales del departamento de Boyacá con código SGI 2879, y financiado por la Universidad Pedagógica y Tecnológica de Colombia. 
Los estudios sobre ambientes de aprendizaje son interdisciplinares y habitualmente son abordados desde perspectivas quedesligan las problemáticas educativas de la institucionalidad, avocando así toda la responsabilidad a la escuela y sus dinámicas. Para evitar caer en generalizaciones, Duarte (2003) asigna a los ambientes de aprendizaje tres ejes de reflexión: lo lúdico, lo estético y lo cultural.

De esta manera, surgen diversos requerimientos para incorporar la escuela a las transformaciones de circulación y producción del saber en las sociedades de la información. Entre las exigencias más sobresalientes se encuentran la reflexión sobre el proyecto de sujeto y las formas de relación e interacción en la comunidad educativa, espacios físicos, recursos, y materiales. Teniendo en cuenta esto, la escuela y sus actores deben vincularse a las transformaciones culturales, políticas, económicas y de relación con el saber, en contexto micro y macrosocial, lo cual involucra la dimensión afectiva de las personas. En consecuencia, la escuela ha de ser un espacio donde se piense y se viva en y para la democracia (Duarte, 2003).

A diferencia de otros escenarios de aprendizaje, la escuela se constituye en un proyecto intencionado de formación de sujetos, en este sentido, debido a la mediación y avance de las nuevas tecnologías de la comunicación, el acceso a los saberes ya no es un tema intergeneracional. Gracias a la relativización de los tiempos, espacios y medios para el aprendizaje, la culturización de los sujetos no depende exclusivamente de una formación intergeneracional, en vez de ello, está sujeta al consumo de información.

En consecuencia, las instituciones educativas deben garantizar el acceso al aparato tecnológico, pues si lo limita, más que aportar al proceso de aprendizaje obstruirá el acceso al saber, o al menos a la información, en tanto, el aparato tecnológico con que circula el saber se configura como un lugar de construcción de conocimiento y de sujeto (Salinas, 1997). Por tanto, es preciso resaltar que la escuela debe transformar sus escenarios, procurar espacios donde la presencialidad y la virtualidad interactúen significativamente y se establezcan como necesidades, en favor de los procesos formativos. La reflexión sobre las interrelaciones establecidas por escenarios virtuales y presenciales es lo que se denomina educación flexible (Duarte, 2003)

En el marco de lo institucional, se entiende que la mediación de las Tecnologías de la Información y Comunicación (TIC) hace que los lugares donde se aprende sean relativos (Pulido et al., 2016). Aunque las TIC abren posibilidades para el acceso a la información, también se constituyen como lugares de exclusión y establecen una delgada línea entre quienes poseen y

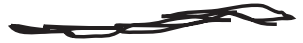

"A diferencia de otros escenarios de aprendizaje, la escuela se constituye en un proyecto intencionado de formación de sujetos" 
dominan herramientas informáticas, y aquellos que carecen de ellas, lo cual puede conllevar al analfabetismo de esa población en términos tecnológicos.

Así mismo, la inclusión de las TIC en la escuela representa la inclinación hacia una mimesis de los colegios con factores externos a la institución. Pero tal vez, en este esfuerzo bien intencionado por redimir a la escuela, se niega la posibilidad de convertirla también en un lugar de suspensión de lo que se presenta como natural en la vida social:

Lo escolar también hace referencia, junto con la suspensión, a la creación de nuevos espacios y tiempos que son posibilidades para la libertad; espacio y tiempo ocupados por todos y cada uno, que producen escenas y formas de reapropiación, que a su vez se convierten en públicas, formas dispuestas para el acceso a la crítica, la confrontación y la innovación. (Pulido, 2018, p. 10).

\section{Sobre la filosofía y la comunidad de indagación}

La filosofía en el aula se entiende como la posibilidad para explorar y fomentar habilidades de pensamiento a través del diálogo. Se espera que, al llevar la filosofía al aula como una actitud frente a la existencia, los estudiantes sean capaces de identificar herramientas que les permitan desarrollar su pensamiento y capacidad argumentativa dentro y fuera del espacio escolar.

En este sentido, Mathew Lipman propone un programa que involucra el desarrollo del pensamiento filosófico desde la infancia. Este planteamiento ha tenido diversos lugares de aplicación y apropiación, por ejemplo, Jorge Iván García (2011) da cuenta de las potencialidades de la propuesta Filosofía para niños, en el mejoramiento de las relaciones dialógicas de estudiantes de cuarto primaria, expresadas en la preocupación de los niños y niñas por dar cada vez mejores razones que sustenten sus argumentos en medio de una discusión. Igualmente, el documento: La Filosofía, una escuela de la libertad. (UNESCO, 2011), evidencia las diversas apropiaciones del proyecto original de Lipman en varios escenarios escolares y países del mundo.

Así mismo, es relevante destacar que la propuesta inicial de Lipman centrada en Filosofía para Niños (FpN), pasa a reformularse como Filosofía con Niños $(\mathrm{FcN})$ a causa de investigaciones desarrolladas por autores como Diego Antonio Pineda (2007; 2009; 2015), Olga Grau (2006), Eugenio Echeverría (2014), Anna Marí Vicuña y Walter Omar Kohan (Kohan, 2004; 2007; 2008; 2009; 2011), María Teresa Suárez y colaboradores (2017), y Oscar Pulido (Espinel \& Pulido, 2017; Pulido, 2009), entre otros, en el contexto latinoamericano. Estas reformulaciones no deslegitiman la propuesta inicial, 
más bien, la enriquecen al contemplar diversas miradas epistemológicas sobre la educación y la infancia, y el lugar de la filosofía en ellas. Del mismo modo, en los trabajos descritos, el desarrollo de la comunidad de indagación ocupa un lugar primordial y se hace manifiesta la intención de desarrollar la educación filosófica como concepto, más allá de pensar simplemente las formas de una clase de filosofía o ética.

El aula como comunidad de diálogo e indagación es una práctica relativamente nueva que requiere formas de interacción y relación entre maestro y estudiantes particulares. La visión tradicional del maestro en la cúspide de las relaciones escolares debe movilizarse hasta encontrar formas de relación menos verticales que propicien espacios de respeto, de esta manera, se pasa a formas de autoridad relativa en la que la voz y el actuar del estudiante se abren espacio como ejes fundamentales a la hora de los ejercicios en el aula. En consecuencia, para el desarrollo de conciencia sobre el trabajo en equipo, se hace necesario fomentar las potencialidades y habilidades en el diálogo para ello se entablan nuevas relaciones donde se deja atrás la recitación memorística de información y se da paso a la co-construcción dialógica (Wells \& Mejía-Arauz, 2005). Estos cambios, se propician en una comunidad preocupada por indagar, más que por dar por hechos ciertos conocimientos.

La comunidad que acá se propone, es guiada por una perspectiva de indagación en la que los sujetos involucrados son capaces de hacer explícitas sus preocupaciones y ponerlas en discusión con los demás, de tal manera que el ejercicio implica poner los conocimientos en suspensión, para ser analizados, criticados, discutidos y recreados (Cerletti, 2008). En este sentido, el pensar y aplicar la comunidad de indagación como un ambiente de aprendizaje se convierte también en un ejercicio democrático (Pineda, 1992). Es entonces el objeto de la comunidad de indagación, propiciar espacios en torno al diálogo con el fin de preparar a los sujetos para una vida en democracia (Almanza, 2017) .

El desarrollo de la globalización y la inserción de las tecnologías en las últimas tres décadas, ha transformado las relaciones espaciales, llevando a nuevas formas de relacionarse con y en lo público, ante lo cual emergen reflexiones sobre el lugar que ocupa lo público en la escuela (EspinozaDiáz \& Arredondo-Ramírez, 2019). Espinoza-Diáz y Arredondo-Ramirez exploran las potencialidades que establece la comunidad de indagación en la construcción de relaciones éticas y políticas en el espacio público que resulta ser la escuela. La indagación en este caso representa la necesidad de cuestionar los principios mismos que establecen lo público, lo ético, lo moral, lo político, etc. La Propuesta de Espinoza y Arredondo (2019) llega a 


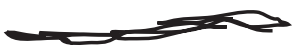

"Se hace referencia entonces, a un rescate de las cargas afectivas en las relaciones que se establecen en los ecosistemas escolares, estableciendo estos escenarios como los

lugares propicios para las discusiones alrededor de problemas éticos" ser plausible bajo determinadas circunstancias curriculares e institucionales.

La incursión de las nuevas tecnologías resulta imperativa para la escuela y las distintas apropiaciones del programa de Filosofía para/con niños y la comunidad de indagación, en este sentido, apropiaciones que apuestan por establecer un modelo de comunidad de indagación mediado por las TIC y pensado desde la idea de los estilos de aprendizaje (Martínez-Bernal et al., 2016). Teniendo en cuenta los postulados de la comunidad de indagación, no se trata de establecer modelos como lo proponen estos tres autores, en vez de ello, se procura reconocer los principios básicos de la comunidad de indagación como el respeto, la disposición del espacio, la búsqueda de preguntas y razones pulidas en el diálogo. Aun así, es de rescatar las intenciones en cuanto a la construcción de determinadas formas de hacer comunidad con la mediación de las TIC rescatando las cargas afectivas (Partida et al., 2015).

Se hace referencia entonces, a un rescate de las cargas afectivas en las relaciones que se establecen en los ecosistemas escolares, estableciendo estos escenarios como los lugares propicios para las discusiones alrededor de problemas éticos. Las dinámicas escolares de sociedades como la colombiana, presentan fuertes tendencias a reducir los espacios para la reflexión ética (Zapata \& Granada, 2017), situación similar sufre la enseñanza de la filosofía. Sin embargo, esto no da lugar a derrotismos, más bien abre la posibilidad de replantear los ambientes que aún perduran en la escuela y de acceder a nuevos como los que propicia la virtualidad (Torras, 2013).

El desarrollo de pensamiento que se propone a partir de Lipman establece tres habilidades que resultan primordiales: el pensamiento crítico, pensamiento cuidadoso y pensamiento creativo (Lipman, 1989). El trabajo realizado por Hernández y Hernández (2012) explora los resultados de ambientes de aprendizaje enfocados en el desarrollo de pensamiento creativo. Es necesario destacar, que la comunidad de indagación abre la posibilidad de poner en discusión los distintos problemas éticos que se vinculan con la vida de los estudiantes, esperando que ello influya en climas escolares más positivos.

No obstante, es bien sabido que para generar cambios en la estructura de las relaciones todo espacio es propicio para tratar y discutir dilemas éticos, por ello, no es una labor exclusiva de las clases de ética y valores humanos. Esto implica un cambio actitudinal de los maestros (Correa, 1998). En este sentido, las principales características de la comunidad de indagación como ambiente de aprendizaje de dilemas éticos, involucran la utilización de un detonante de estas disyuntivas, que bien puede ser una pregunta, un texto, 
un video etc. Es necesario desatacar el papel que juega la disposición del maestro y de la institución misma en estos procesos.

Como propuesta de un modelo en clave de comunidad de indagación, Saavedra (2011) diseña una unidad didáctica en la que se pueden evidenciar cuatro momentos a tener en cuenta para la preparación de una clase. El primero tiene que ver con los elementos detonantes que son escogidos a libertad por los estudiantes o docente, el segundo momento se denomina exploremos, en donde se establecen preguntas que movilicen la discusión y exploración de saberes entre la comunidad. Luego aparece recorriendo senderos, donde se ubican los tiempos concretos de la clase en los cuales los estudiantes leen, analizan, dialogan y producen. Finalmente aparece mis avances, el cual define lo que el docente espera que sea aprendido durante la clase.

\section{Metodología}

El presente es un estudio de caso que, en la perspectiva de Robert Stake (1998), se define como un estudio basado en la particularidad, que no necesariamente contempla la generalización. Sin embargo, puede aportar a esta dependiendo de las dimensiones de análisis del caso, es frecuente observar su uso dirigido hacia el análisis de un caso singular, comprendiendo así actividades en diferentes circunstancias.

Para el análisis de este proyecto se partió de un diagnóstico, que consistió en analizar el uso del observador por parte los docentes que orientan clase a un grupo de estudiantes de grado sexto, para identificar la afinidad normativa por parte de los docentes con un grupo calificado con problemas de comportamiento.

Problemática. Las discusiones alrededor del comportamiento de los estudiantes son frecuentes en la cotidianidad de las instituciones educativas, ya sea porque no es adecuado o no va de acuerdo con las normas establecidas en el manual de convivencia institucional, por ello, docentes y directivos buscan medidas y estrategias que se encaminan a aportar en el mejoramiento comportamental de los estudiantes. La escuela es una representación inicial de la sociedad secundada por la normas del núcleo familiar, se asume entonces, que es en la escuela donde se reproducen a menor escala los andamiajes normativos de la sociedad, la esencia de la convivencia y sus reglas (Bourdieu, 2005). Por ello, es importante que al estudiante se le dé a conocer la base normativa de la institución educativa, expuesta habitualmente en el manual de convivencia, entendido éste como un código de conducta y castigo, así, el estudiante podrá encontrar los límites y consecuencias de sus acciones. 
La Institución donde se desarrolló esta investigación ${ }^{1}$ se identifica con el carácter normativo que representa el manual de convivencia escolar, procurando que sea el punto de partida para cualquier juicio y observación comportamental. El incumplimiento de dicho reglamento se registra sistemáticamente por parte de docentes y directivos en el observador del alumno. Este documento asume las faltas en dos dimensiones: comportamental y académica.

La dimensión comportamental comprende una mayor tipología de las faltas realizadas por los estudiantes, que la dimensión académica. En este sentido se observa que el tipo de falta que destaca es la indisciplina en clase, la cual está enmarcada en la charla poco moderada entre compañeros, jugar en el aula, y desobedecer al docente. Así mismo, aparece como falta la evasión a clase, la cual es registrada cuando se presentan reiteradas impuntualidades por parte de los estudiantes para el ingreso a las clases, habitualmente, por permanecer jugando en el patio del colegio en horarios no permitidos. Igualmente, el irrespeto a los compañeros coexiste junto con la indisciplina, en la medida que hace parte de las conversaciones entre estudiantes en el desarrollo de las clases, generando malestar en el aula. Finalmente, cabe resaltar que tanto el ingreso de armas como el insultar docentes, están calificados como faltas graves que pueden llevar al estudiante a cancelación de matrícula. Sin embargo, el manual contempla otras faltas en las que no ha incurrido el grupo objeto de este estudio.

Las faltas en la dimensión académica están enmarcadas, en primer lugar, por la no realización de deberes académicos por parte de los estudiantes, éstas se registran en el observador del estudiante cuando ocurre de manera reiterada. El juicio que se elabora y consigna en el observador es: no cumple con los criterios de evaluación propuestos por los docentes, es decir, el estudiante no alcanza los criterios de la evaluación establecidos, además, es consecuencia del no trabajo en clase. Finalmente, una falta que podría ser considerada como grave, pero que no se encuentra normalizada en el manual de convivencia es el fraude en las evaluaciones, pues los estudiantes registran dicha falta en repetidas ocasiones, sin embargo, la observación se realiza sin ningún sustento normativo.

En el grupo estudiado, se encuentra que el observador del estudiante suele ser usado con mayor frecuencia por el director de grupo, sin embargo, de las 68 observaciones registradas y analizadas en esta investigación, 64 corresponden a registros sobre la entrega de informes, de manera que solo 4 anotaciones se relacionan con faltas del manual de convivencia como:

Por norma ética, el nombre de la institución educativa se mantiene en anonimato. 
romper un vidrio durante el descanso. Así mismo, el docente de Ciencias Sociales registra 17 observaciones, dentro de las cuales se destacan como tipo de falta: la indisciplina y el incumplimiento académico. En el área de interés de este proyecto: Ética y Valores Humanos, se observa una frecuencia de uso de 12 observaciones, dentro de las cuales, al igual que en el área de sociales, predomina la falta denominada indisciplina como se relaciona en la figura 1.

El anterior diagnóstico crea un juicio sobre el comportamiento de los estudiantes, en el cual se evidencia el incumplimiento de normas establecidas por la institución educativa, en consecuencia, la pregunta a resolver es ¿cómo un ambiente de aprendizaje creado en clave de comunidad de indagación, propia del programa filosofía para/con niños, desarrollado en el área de ética y valores humanos, puede promover un cambio de comportamiento en los estudiantes de grado sexto de la institución educativa objeto de estudio?.

Figura 1.

\section{Uso del observador por docentes}

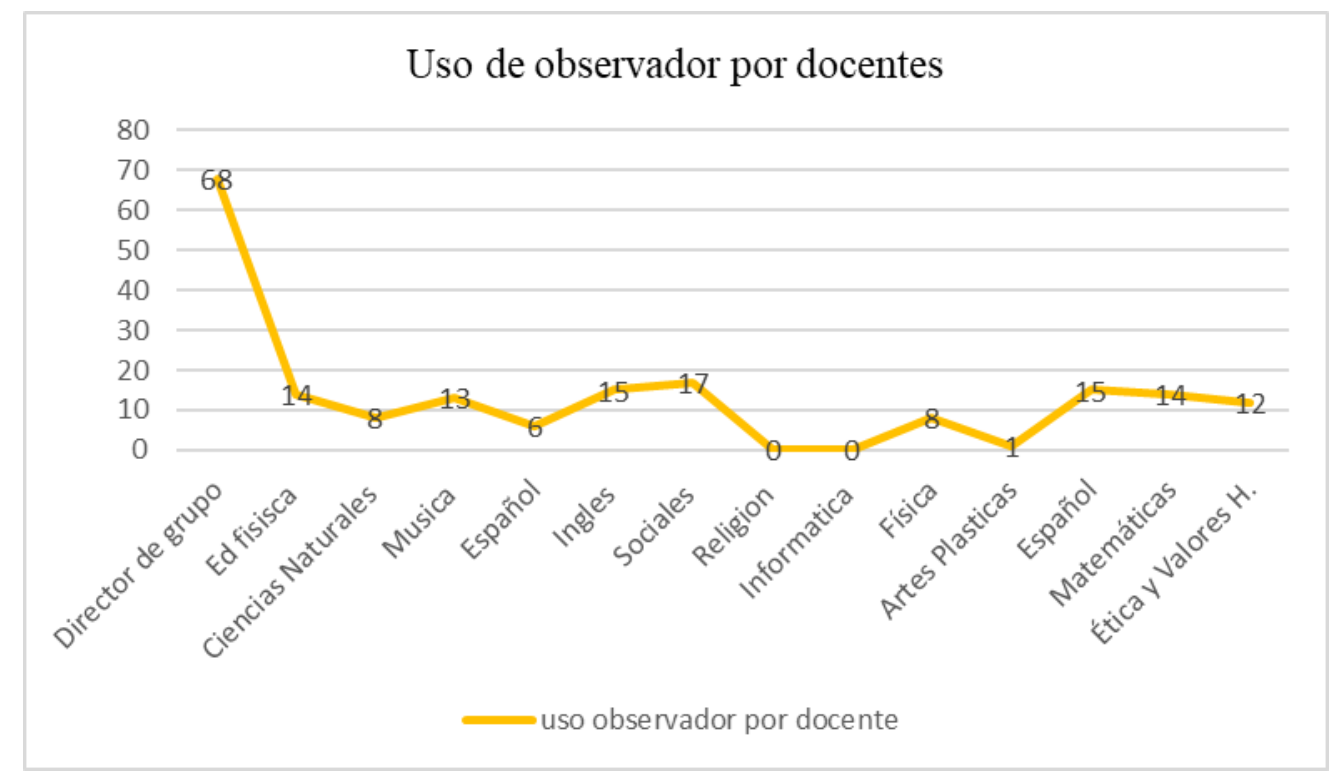

Con el diagnóstico antecedente se procede a la aplicación de un ambiente de aprendizaje creado en clave comunidad de indagación, propia del programa filosofía para niños, el cual se desarrolló en el área de Ética y Valores Humanos. Tomando como referencia el modelo de unidad didáctica propuesto por Saavedra (2011), el cual contempla cuatro momentos a tener en cuenta en la preparación de una clase en clave comunidad de indagación, se diseñaron los lugares para el ambiente de aprendizaje, los cuales fueron construidos desde las temáticas establecidas en la planeación de área institucional.
"Con el diagnóstico antecedente se procede a la aplicación de un ambiente de aprendizaje creado en clave comunidad de indagación" 


\section{Resultados de la evaluación del ambiente de aprendizaje}

A continuación, se presentan los resultados de la evaluación realizada por estudiantes respecto del ambiente de aprendizaje aplicado. Ésta, utiliza una escala de Likert (Nunca, Casi nunca, Casi siempre y Siempre) que permite valorar los criterios y categorías a evaluar, los cuales son:

- Conocimientos previos: Las preguntas de esta categoría pretenden dar cuenta de la percepción sobre cómo el maestro tiene en cuenta antes, durante y después de las sesiones de clase los conocimientos que dominan los estudiantes

- Ambiente de seguridad, respeto y confianza: Se espera evidenciar la apreciación de los estudiantes respecto a la calidad de las relaciones que se dan en el desarrollo de la clase

- Diseño y ejecución de actividades retadoras e innovadoras: Se busca dar cuenta de las actividades que se establecen como retos motivantes de la discusión y el dialogo.

- Estrategias, recursos y momentos de evaluación: Se construye desde la fuerza de apropiación teórica del profesor y las cargas no declaradas del sistema de creencias.

- Infraestructura y materiales: Se espera evidenciar la percepción de los estudiantes en cuanto a la recursividad del maestro para hacer uso de los espacios y materiales con que cuenta la institución, para el desarrollo de la sesión formativa

- Socialización de aprendizajes a la comunidad: Se espera dar cuenta de la comunicación que se da luego de la sesión de clase con la comunidad educativa.

Figura 2

Resultados de la evaluación, categoría: conocimientos previos

\section{Conocimientos previos}

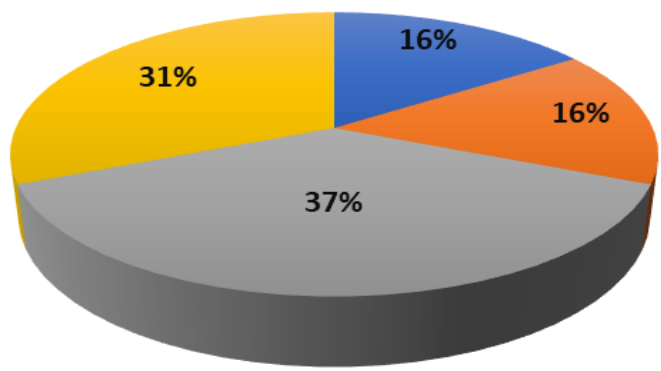

Eunca En algunas ocasiones a Casi siempre 
En cuanto a si la clase se considera como un espacio y ejercicio en el que se reconocen los conocimientos previos que los estudiantes demuestran, éstos consideran, de acuerdo con los resultados de la evaluación, que la docente siempre o casi siempre los tiene en cuenta para impartir su clase (Figura 2). En este sentido, la maestra recurre a instrumentos como cuestionarios, socializando en antes, durante y después de la clase, el tema a tratar.

La figura 3 muestra los resultados en cuanto a la percepción de ambiente de seguridad, respeto y confianza, en este sentido, se evidencia una apreciación positiva por parte de los estudiantes en cuanto al respeto durante el desarrollo de la clase. No obstante, es necesario resaltar la también significativa apreciación de aquellos que consideran que no se cumple con unos parámetros básicos de respeto en el desarrollo de la clase. Esta disparidad en la apreciación del ambiente del aula puede deberse a los recurrentes momentos de los que podría parecer desorden al momento de participar en el dialogo alrededor del tema tratado. Aun así, estas valoraciones nos permiten deducir que la docente se interesa por la participación de los estudiantes dando lugar a momentos de discusión más abiertos y fluidos.

Figura 3

Resultados de la evaluación, categoría: ambiente de seguridad respeto y confianza

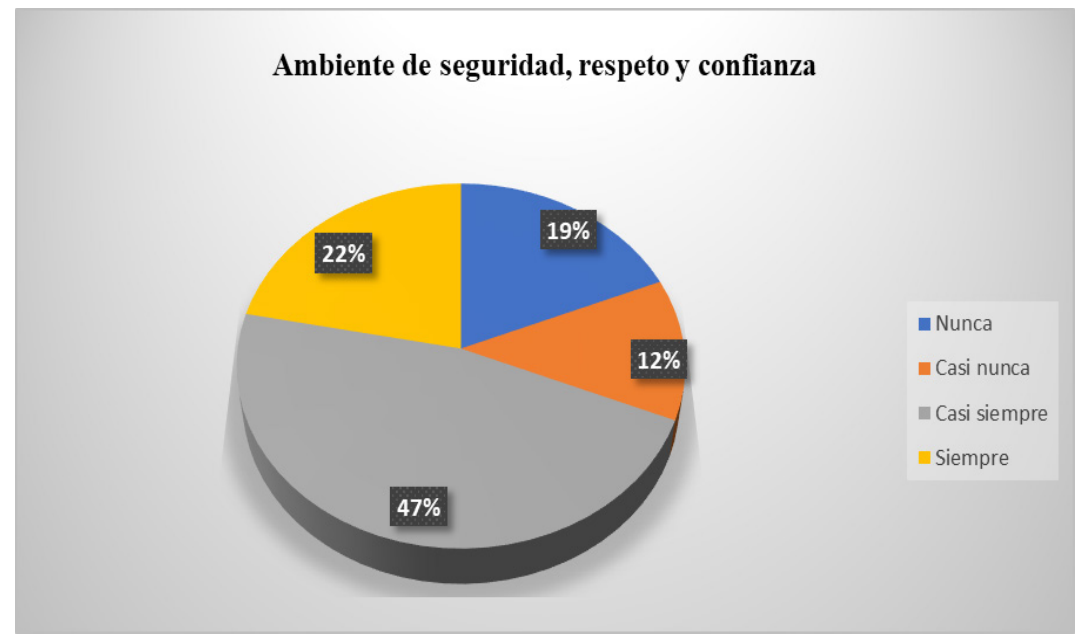

Es importante resaltar el lugar que la docente le otorga a la pregunta en su clase, pues a través del interrogante se establecen retos de discusión que permiten movilizar la temática a lo largo de la sesión. La opinión de los estudiantes frente a este ítem (Figura 4) deja ver que la docente se empeña por hacer búsqueda e indagación con relación a la temática que moviliza, recurriendo a referentes teóricos y a ejemplos prácticos. 
Figura 4

Resultados de la evaluación categoría: Diseño y ejecución de actividades retadoras e innovadoras

\section{Diseño y ejecucion de actividades retadoras e innovadoras}

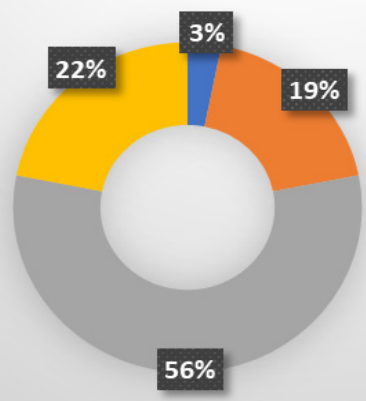

- Nunca

Casi nunca

- Siempre

- Casi siempre

La figura 5 detalla la percepción de los estudiantes respecto a las estrategias, recursos y momentos de evaluación en clase, en este sentido, se evidencia que un $47 \%$ reconoce que la docente casi siempre evalúa a lo largo del desarrollo de la sesión. No obstante, también es significativo el porcentaje de opinión de quienes consideran que la maestra no recurre a procesos ni estrategias de evaluación en los tres momentos de la clase.

Figura 5

Resultados de la evaluación, categoría: Estrategias, recursos y momentos de evaluación.

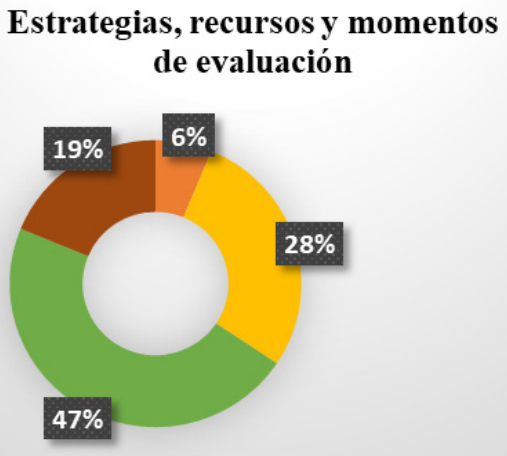

Nunca

Casi Nunca

Casi siempre

- Siempre

Dado que la sesión de clase se dio de manera tradicional en el aula, la percepción de los estudiantes en cuanto al uso y aprovechamiento de los recursos e infraestructura de la institución, es baja (figura 6). Esto puede exponer la necesidad de dar lugar al desarrollo de las sesiones en espacios distintos al aula. Ahora bien, las condiciones mismas de la institución y algunas normativas propias impiden que estas dinámicas sean ejecutadas, no 
obstante, los estudiantes manifiestan su interés por hacerlo.

Figura 6

Resultados de la evaluación, categoría: infraestructura y materiales

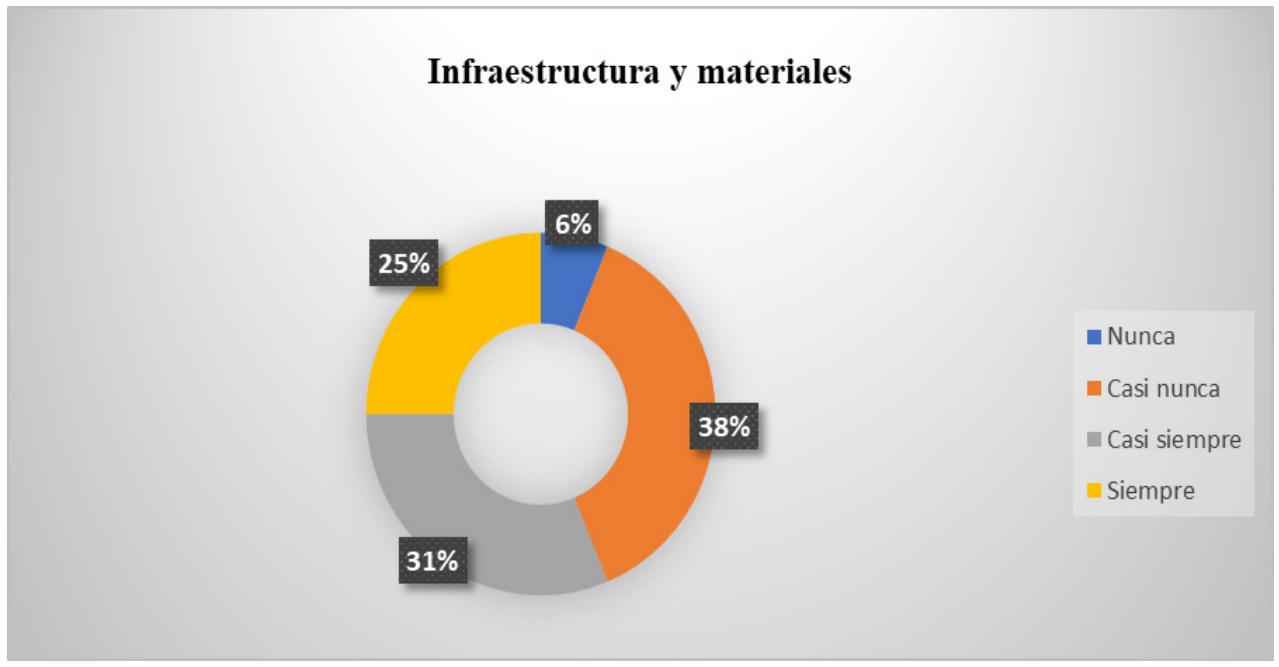

Finalmente, las apreciaciones de los estudiantes en cuanto a cómo la docente propicia espacios de diálogo y socialización tienden a considerar que la mayoría de las veces se promueve la socialización de los aprendizajes logrados en el grupo (Figura 7). No obstante, hace falta establecer estrategias que permitan una comunicación directa con la comunidad educativa en relación a los aprendizajes alcanzados en la clase de ética.

Figura 7

Resultados de la evaluación, categoría: Socialización de aprendizajes a la comunidad

\section{Socializacion de aprendizajes a la comunidad}

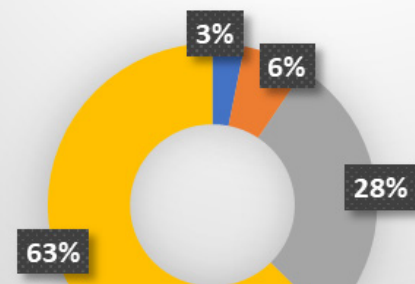

$$
\begin{aligned}
& \text { Nunca } \\
& \text { Casi nunca } \\
& \text { Casi siempre } \\
& \text { Siempre }
\end{aligned}
$$




\section{Conclusiones}

Basados en la evaluación realizada por parte de los estudiantes al ambiente de aprendizaje aplicado, se evidencia una alta favorabilidad y aceptación de éste. Lo apreciaron como la posibilidad de explorar otras maneras de aprender y hacerse conscientes de su propio estilo de aprendizaje, además de reconocer la importancia de escuchar al otro y respetarlo, especialmente en los espacios formativos. La comunidad de aprendizaje permitió que los estudiantes descubrieran el lugar que cada uno ocupa a través del uso de la palabra, estableciendo lazos de respeto y escucha que incluso propició que compartieran anécdotas.

Así mismo, la aplicación de la unidad didáctica basada en la apropiación del programa filosofia para niños, es completamente viable metodológicamente pues en el aula se puede llevar a cabo su desarrollo de manera intuitiva. Permite explorar saberes previos e inquietarse por lo que se desconoce y de esta manera apropiar saberes nuevos.

Frente a las transformaciones comportamentales con la aplicación del ambiente de aprendizaje, se puede indicar que estas siempre sucedieron en mayor o menor grado en cada estudiante, sin embargo, este es un proceso que debe ser evaluado a largo plazo, pues implica trabajar de manera conjunta y paralela las tres dimensiones del pensamiento complejo: la crítica, la cuidadosa y la creativa, resulta fundamental resaltar que, la convergencia de estas tres dimensiones no aporta únicamente al aprendizaje de la ética, también repercute en cualquier asignatura en el caso de la escuela secundaria o primaria, o disciplina para el caso de la educación superior.

Igualmente, se desataca que las distintas formas que ha tomado la propuesta inicial de Lipman responden a las variables que ofrecen los contextos a donde es llevada, pues las apropiaciones generan adaptaciones de implementación de acuerdo al entorno. En algunos lugares la aplicación de las novelas y los manuales de Lipman se dan agracias a individualidades de los maestros, y en otros lugares, el currículo se ha transformado teniendo en cuenta las distintas concepciones del trabajo filosófico con niños, niñas y adolescentes. Es en estos últimos lugares donde la aplicación de $\mathrm{FpN}$ o $\mathrm{FcN}$ tiene una mayor trascendencia, al apostar por una educación filosófica que involucra asumir la filosofía como una forma de vida o una actitud de pensamiento (Bohórquez, 2016), por lo que transgrede la idea de una catedra de filosofía para plantear formas de enseñar y aprender indistintamente del campo de saber o disciplina, ya que lo que se apuesta es a formar y potenciar una actitud hacia el mundo práctico y de saberes (García \& Martínez, 2015).

En consecuencia, las distintas propuestas y apropiaciones de FpN y FcN apuntan a la transformación educativa entendida como una comunidad que 
investiga para enseñar y aprender. En consonancia con esta perspectiva, la escuela debe reformular los principios éticos y morales sobre los que se funda; ha de suspender la estereotipación de formas correctas de comportamiento y la idea de la escuela como una copia a escala de la sociedad. La escuela como comunidad de indagación no impone comportamientos ni formas de hacer sociedad ya que la comunidad misma se asume, al igual que los conocimientos y saberes, como algo inacabado, lo que le permite suspender lo que pasa fuera de la escuela para experimentar nuevas formas de relación (Pulido, 2018). Aun así, la comunidad no parte de un relativismo total, sino que contempla la necesidad de la preservación del otro como forma de la conservación propia, es decir, de la necesidad de cuidar del otro y de lo otro como la única forma de garantizar el propio cuidado.

\section{Referencias}

Almanza, M. (2017). La implementación de una comunidad de indagación al estilo de Filosofía para Niños y la posibilidad de construir una identidad democrática en los niños de grado sexto del colegio General Gustavo Rojas. [Trabajo de maestría, Universidad Santo Tomás]. https://doi.org/10.15332/tg.mae.2017.00311

Bohórquez, I. S. (2016). El espíritu científico en la primera infancia. Praxis \& Saber, 7(13), 89-106. https://doi.org/10.19053/22160159.4167

Bourdieu, P. (2005). Capital cultural, escuela y espacio social. Siglo XXI.

Hernández, A., \& Hernández, M. (2012). Ecosistemas escolares para el desarrollo del pensamiento creativo en los niños. Praxis \& Saber, 3(6), 141-164. https://doi.org/10.19053/22160159.2007

Cerletti, A. (2009). La enseñanza de la filosofía como problema filosófico. Libros el Zorzal.

Correa, A. G. (1998). Un aula pacífica para una cultura de paz. Revista electrónica interuniversitaria de formación del profesorado, 1(1), 1-16.

Duarte, J. (2003). Ambientes de aprendizaje: una aproximación conceptual. Estudios pedagógicos, (29), 97-113. https://doi.org/10.4067/S071807052003000100007

Echeverría, E. (2014). La comunidad de diálogo filosófico como estrategia para el apoyo a niños y adolescentes migrantes [Ponencia]. VII Encuentro Filosofía e Infancia. Tunja, Universidad Pedagógica y Tecnológica de Colombia.

Espinel, Ó., \& Pulido, Ó. (2017). Enseñanza de la filosofía. Entre experiencia filosófica y ensayo. Universitas Philosophica, 34(69), 121-142. https://doi. org/10.11144/Javeriana.uph34-69.efee 
Espinoza-Díaz, C., \& Arredondo-Ramírez, C. (2019). El rol de la comunidad de indagación en el espacio público escolar. Praxis \& Saber, 10(23), 77-96. https://doi.org/10.19053/22160159.v10.n23.2019.9693

García., J. I. (2011). Cómo la propuesta de filosofía para niños incide en el diálogo filosófico de los niños y niñas del grado 4 de la I.E. Augusto Zuluaga Patiño. [Tesis de maestría, Universidad Tecnológica de Pereira]. http://repositorio.utp.edu.co/dspace/ handle/11059/2623

García, N., \& Martínez, L. (2015). Incidencia del abordaje de una cuestión sociocientíficaen la alfabetización científicay tecnológica dejóvenesy adultos.Praxis \& Saber, 6(11), 87 - 114. https://doi.org/10.19053/22160159.3576

Grau, O. (2006). Filosofía para la infancia: Relatos y desarrollo de actividades. Buenos Aires: Noveduc Libros.

Pulido, D., Nájar, O., \& Guesguan, L. (2016). Vivamos la innovación de la inclusión de dispositivos móviles en la educación. Praxis \& Saber, 7(14), 115 - 140. https://doi.org/10.19053/22160159.5220

Kohan, W. (2004). Infancia: Entre educación y filosofía. Barcelona: Laertes.

Kohan, W. (2007). Infancia, politica y pensamiento. Argentina: Del estante.

Kohan, W. (2008). Filosofía, la paradoja de aprender y enseñar. Libros del Zorzal.

Kohan, W. (2009). Acerca de un motivo infantil para filosofar. Revista internacional Magisterio, 2 (21).

Kohan, W. (2011). Filosofía y educación. La infancia y la política como pretextos. Fundarte.

Lipman, M. (1989). Pensamiento complejo y educación. Ediciones de la Torre.

Martínez-Bernal, J., Sanabria-Rodríguez, L. B., \& López-Vargas, O. (2016). Relaciones entre logro de aprendizaje, automonitoreo, estilo cognitivo y estilos de aprendizaje en estudiantes de medicina. Praxis \& Saber, 7(14), 141164. https://doi.org/10.19053/22160159.5221

Partida, S. P., Vera, L. J. O., \& Madrid, C. L. (2015). Comunidades de aprendizaje en línea Análisis de las interacciones cognitivas, docentes y afectivas. Apertura, 7(1), 108-127.

Pineda, D. (1992). Filosofía para niños: Un acercamiento. Universitas Philosophica, 10(19), 103-121.

Pineda, D. (2007). Hacia una reflexión filosófica por medio de la investigación del crimen: Una experiencia de pedagogía filosófica a partir de Sherlock Holmes. Cuestiones de Filosofía, 9, 109-142. 
Pineda, D. (2009). Propuesta de educación moral desde una perspectiva filosófica para la escuela primaria. Cuestiones de Filosofía, 11, 2-23.

Pineda, D. (2015). Apuntes para la comprensión de «El descubrimiento de Harry», de Matthew Lipman. Análisis, 47(86), 21-68. https://doi. org/10.15332/s0120-8454.2015.0086.02

Pulido, Ó. (2009). Aprender y enseñar filosofía en el mundo contemporáneo: De la mercantilización del pensamiento al despliegue de su ejercicio. Cuestiones de Filosofía, 11. 87-103. https://doi.org/10.19053/01235095.653

Pulido, Ó. (2018). ¡Hay que defender la escuela!. Praxis \& Saber, 9(20), 9-14. https://doi.org/10.19053/22160159.v9.n20.2018.8426

Saavedra,M.(2011).Delaula declasetradicionalala comunidad deinvestigación. Praxis \& Saber, 2(4), 179-200. https://doi.org/10.19053/22160159.1121

Salinas, J. (1.997). Nuevos ambientes de aprendizaje para una sociedad de la información. Pensamiento Educativo, 20 (1), 81-104.

Stake, R. E. (1998). Investigación con estudio de casos. Ediciones Morata.

Suarez, M. T., González, B. A., \& Lara, P. (2017). Apropiaciones y experiencias pedagógicas de filosofía e infancia en Colombia. Praxis \& Saber, 8(16), 225-247. https://doi.org/10.19053/22160159.v8.n16.2017.6184

Torras, V. (2013). Ambientes virtuales de aprendizaje en la etica. [Presentación en Slide Share]. https://es.slideshare.net/claudioclarenc/ambientesvirtuales-de-aprendizaje-en-la-etica

UNESCO, O. (2011). La Filosofía, una escuela de la libertad. UNESCO.

Wells, G., \& Mejía-Arauz, R. (2005). Hacia el diálogo en el salón de clases: Enseñanza y aprendizaje por medio de la indagación. Sinéctica, Revista Electrónica de Educación, (26), 1-19.

Zapata, \& Granada (2017). La ética en los ambientes educativos: ¿un tema minusvalorado en la educación del siglo XXI? Revista Reflexiones y Saberes, 4(6), 28-39. 